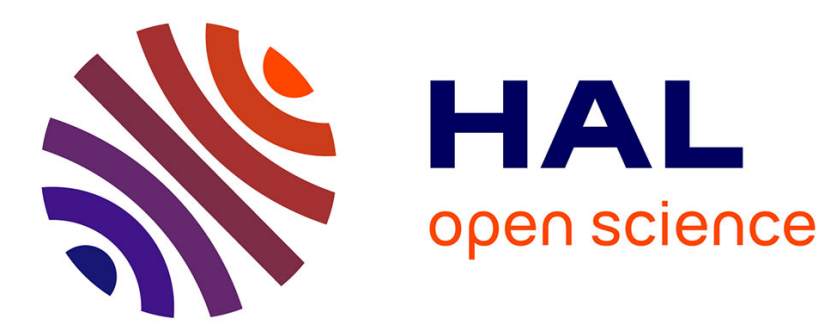

\title{
Effect of cross-links on a mixture of polymers
}

P.-G. de Gennes

\section{To cite this version:}

P.-G. de Gennes. Effect of cross-links on a mixture of polymers. Journal de Physique Lettres, 1979, 40 (4), pp.69-72. 10.1051/jphyslet:0197900400406900 . jpa-00231571

\section{HAL Id: jpa-00231571 https://hal.science/jpa-00231571}

Submitted on 1 Jan 1979

HAL is a multi-disciplinary open access archive for the deposit and dissemination of scientific research documents, whether they are published or not. The documents may come from teaching and research institutions in France or abroad, or from public or private research centers.
L'archive ouverte pluridisciplinaire HAL, est destinée au dépôt et à la diffusion de documents scientifiques de niveau recherche, publiés ou non, émanant des établissements d'enseignement et de recherche français ou étrangers, des laboratoires publics ou privés. 


\title{
Effect of cross-links on a mixture of polymers
}

\author{
P. G. de Gennes \\ Collège de France, 75231 Paris Cedex 05, France \\ (Reçu le 28 novembre 1978, accepté le 21 décembre 1978)
}

\begin{abstract}
Résumé. - Nous considérons un mélange liquide de deux polymères (A et B) légèrement incompatibles. Ce mélange est supposé réticulé à haute température (ou dans d'autres conditions favorisant la miscibilité dans l'état initial). Après réticulation, la température est abaissée, et les deux types de chaînes tendent à segreger, mais sont maintenues ensemble par les points d'attache $\mathrm{AB}$. Nous prédisons ici l'apparition d'une " microphase ", dans laquelle les concentrations locales en A (et B) jouent le rôle de densités de charge (+ et -). Il n'y a pas de charge macroscopique, mais une polarisation spontanée apparaît pour $T<T_{\mathrm{c}}$. Nous donnons une estimation de $T_{\mathrm{c}}$ (dans un modèle de Flory-Huggins) en fonction du taux de réticulation.
\end{abstract}

\begin{abstract}
We consider a liquid mixture of two weakly incompatible polymers A and B, and assume that this melt is cross-linked at high temperatures (or in other conditions allowing for good miscibility in the initial state). After cross-linking, the temperature is lowered, and the two types of chains would like to segregate, but are kept together by the cross-links. We then predict a novel transition, where the concentrations of A (and B) play the role of a positive (negative) charge density = there is no macroscopic charge, but a spontaneous polarization appears below a certain temperature $T_{\mathrm{c}}$. We give a simplified formula for $T_{\mathrm{c}}$ in terms of the Flory interaction parameter $\chi$ and of the average number of monomers between cross-links.
\end{abstract}

1. Introduction. - Two chemically different polymers $\mathbf{A}$ and $\mathbf{B}$ are usually not compatible in the molten state. In a Flory-Huggins picture [1]. [2], this trend is described by a free energy $F$ (function of the volume fraction $\varphi$ of polymer) of the classical form :

$$
\begin{aligned}
\frac{F}{k T}=N^{-1}[\varphi \ln \varphi+(1-\varphi) \ln (1 & -\varphi)]+ \\
& +\chi \varphi(1-\varphi)
\end{aligned}
$$

where the interaction parameter $\chi$ is positive. [In eq. (1), we have chosen for simplicity a symmetrical case, where the degree of polymerization $N$, and the volume per monomer $W$ are the same for $\mathrm{A}$ and $\mathrm{B} . F$ is the free energy of mixing per volume $W$.]

A standard discussion of eq. (1) [4] shows that phase separation may occur when $\chi$ is larger than a certain critical value

$$
\chi_{0}=2 N^{-1} \text {. }
$$

The resulting phase diagram is shown qualitatively on figure 1 .

We shall be concerned here with a more complex physical situation :

a) Starting from a melt, described by point $P$ in the one phase region of the diagram, we cross link the

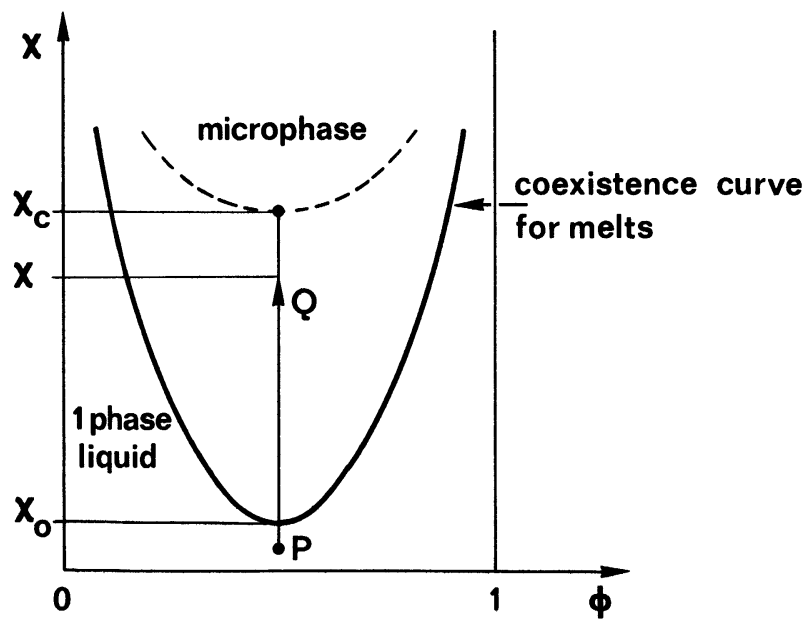

Fig. 1. - Phase diagram for a melt of two polymers AB. $\varphi$ is the volume function of $\mathrm{A}$, and $\chi$ is the Flory interaction parameter. The process discussed in the text corresponds to reticulation at point $P$, followed by an increase in $\chi$, leading to point $Q$. If $Q$ is below the dashed curve, the result is a single phase network. Above the dashed curve, microphase separation occurs.

chains at random : the A chains become attached to the $B$ chains. (On the other hand, the existence, or absence, of A-A and B-B cross-links is not essential. Here we shall assume that only A-B cross-links are present.) We shall specify the level of cross-linking 
through a parameter $n$, giving the average number of monomers between cross-links on one chain. We shall be concerned here with situations well above the gelation threshold, where $n$ is significantly smaller than $N$.

b) After cross-linking, we increase the parameter $\chi$ (for instance through a decrease in temperature) and reach point $Q$ on the phase diagram (Fig. 1). At this stage the chains would like to segregate, but this trend is clearly counteracted by the reticulation. Do we then expect a phase transition, or not ?

This question appears to have some practical interest : polymer blends often allow certain material properties to be optimized; on the other hand, segregation effects may prevent their use. After stage $(b)$ in the above process, if a phase transition takes place, it may lower some material properties (transparency) but it may also improve some other qualities (mechanical behaviour of the composite type).

In the present note, we consider only the existence of a phase transition : we predict a novel type of microphase separation, where small regions become alternatively more concentrated in A or in B. But we do not achieve a complete description of the microphase structure : this would require much more elaborate calculations.

To make the analysis as simple as possible, we choose equal concentrations $\varphi_{A}=\varphi_{B}=\frac{1}{2}$ for the point $\mathrm{Q}$ under study; also our description of the elastic properties is quite primitive (but sufficient for the present purposes).

2. Analogy with a dielectric medium. - In the network, the average concentrations in the sample are fixed, but we can have local fluctuations

$$
\begin{aligned}
& \varphi_{\mathbf{A}}(\mathbf{r})=\frac{1}{2}[1+\rho(\mathbf{r})] \\
& \varphi_{\mathbf{B}}(\mathbf{r})=\frac{1}{2}[1-\rho(\mathbf{r})] .
\end{aligned}
$$

It is convenient to think of $\rho$ as an electric charge density. The macroscopic constraint $\bar{\varphi}_{\mathrm{A}}=\bar{\varphi}_{\mathrm{B}}=\frac{1}{2}$ is the analog of a condition of electric neutrality.

In a dielectric, the + and - charges are tied together, but they can be displaced slightly : a certain polarization $\mathbf{P}$ appears.

$$
\mathbf{P}=\Omega^{-1}\left(\sum_{\mathbf{A}} \mathbf{r}_{i}-\sum_{\mathbf{B}} \mathbf{r}_{i}\right)
$$

where $\mathbf{r}_{i}$ is the position of the (i)th monomer, and where the sums are restricted to a small volume element $\Omega$. Just as in the electrostatic case, polarization and charge are related by the constraint

$$
\operatorname{div} \mathbf{P}=-\rho .
$$

Let us now construct a free energy functional for the network, in terms of the variables $\mathbf{P}$ and $\rho$. This will contain a part dependent on $\mathbf{P}$ and a part dependent on $\rho$, plus cross terms. a) The $P$ part is

$$
\frac{\tilde{F}_{P}}{k T}=\frac{1}{2} C P^{2}+O\left(P^{4}\right) .
$$

Here $C$ is a coefficient of internal rigidity, for which a rough estimate is given in the appendix :

$$
C \cong \frac{36}{n^{2} a^{2}}
$$

The quantity $n a^{2}$ is the mean square end to end distance of a chain of $n$ units. (Again we restrict our attention to the symmetric case, where the A and B chains have the same length parameter $a$.)

Note that in eq. (6) we do not expect terms of odd order in $\mathbf{P}$, because the original network is isotropic : the states $(\mathbf{P})$ and $(-\mathbf{P})$ must have equal free energies.

b) The part of the free energy which depends on $\rho$, $(\widetilde{G}(\rho))$ reflects the trend towards segregation and is directly related to eq. (1).

Expanding eq. (1) around $\varphi=\frac{1}{2}$ we get a first contribution to $\tilde{G}$ :

$$
\frac{1}{k T} \tilde{G}_{1}(\rho)=\frac{1}{4}\left(\chi_{0}-\chi\right) \rho^{2}+O\left(\rho^{4}\right) .
$$

Another term allowing for spatial variations of $\rho$ must also be included : this has been derived in refs. [2] and [4], and is (for slow variations) :

$$
\frac{1}{k T} \widetilde{G}_{2}(\rho)=\frac{a^{2}}{48}(\nabla \rho)^{2} .
$$

c) There exist of course certain cross-terms such as $P^{2} \rho^{2}$ but they can be shown to be unessential for our discussion of the onset of the microphase.

Thus, to second order in $P$ and $\rho$, the total free energy of the cross-linked state $\tilde{F}$ is the sum of eqs. $(6,8,9)$.

3. Onset of a microphase structure. - We now write down the free energy $\tilde{F}$ in terms of Fourier components $\rho_{q}$ and $\mathbf{P}_{q}$. These components are linked by an equation equivalent to (5), namely $i \mathbf{q} \cdot \mathbf{P}_{q}=-\rho_{q}$. We then find that only the component of $\mathbf{P}_{q}$ which is longitudinal (parallel to $\mathbf{q}$ ) can show an instability. Restricting our attention to this component, or equivalently to $\rho_{q}$, we can write :

$$
\begin{aligned}
\frac{1}{k T} \tilde{F} & \rightarrow \sum_{q} \frac{1}{2}\left|\rho_{q}\right|^{2}\left\{\frac{C}{q^{2}}+\frac{1}{2}\left(\chi_{0}-\chi\right)+\frac{a^{2}}{24} q^{2}\right\} \\
& =\sum_{q} \frac{1}{2}\left|\rho_{q}\right|^{2} F(q) .
\end{aligned}
$$

The function $F(q)$ is directly related to the $\mathrm{X}$ ray scattering intensity $I(q)$ at a wave vector $q$

$$
I(q)=\left\langle\left|\rho_{q}\right|^{2}\right\rangle=\frac{1}{F(q)} .
$$


a) When $\chi$ is not much larger than $\chi_{0}, F(q)$ is always positive and has a minimum at a certain wave vector $q^{*}$

$$
\begin{aligned}
& \left(q^{*} a\right)^{4}=24 C a^{2} \\
& \left(q^{*} a\right)^{2}=12 \sqrt{6} n^{-1} .
\end{aligned}
$$

We expect a diffraction peak for $q=q^{*}$.

b) When $\chi$ reaches a critical value $\chi_{\mathrm{c}}$ the factor $F\left(q^{*}\right)$ becomes equal to zero, and this describes an instability. The corresponding value of $\chi,\left(\chi_{c}\right)$ is given by :

$$
\chi_{c}-\chi_{0}=4 C\left(q^{*}\right)^{-2}=\frac{2 \sqrt{6}}{n} .
$$

If we assume that the Flory-Huggins model holds strictly, the interaction parameter $\chi$ is inversely proportional to $T$, and eq. (14) may be written in terms of an instability temperature

$$
T_{\mathrm{c}}=\frac{T_{0}}{1+\sqrt{6} N / n} .
$$

The coefficient $\sqrt{6}$ in this formule is not very precise, for two reasons :

a) our calculation of $C$ is very approximate ;

b) the value of $q^{*}$ given by eq. (13) is not small, as would be required by our use of a gradient expansion in eq. (9).

But the qualitative features of eqs. (13) and (15) should be correct.

4. Discussion. - 1) We see from (15) that a strong cross-linking $(n \ll N)$ increases the region of compatibility by a large factor $\left(T_{\mathrm{c}} \ll T_{0}\right)$. On the other hand, for weak cross-linking $(n \sim N)$, eq. (15) is not good : we need a very different analysis, taking into account the correct scaling laws for the rigidity coefficient $C$ near the sol gel transition.

2) What is the nature of the low temperature microphase ? Eq. (13) tells us that the dimensions of the correlated regions are of order $n^{1 / 2} a$, i.e. comparable to the size of a coil portion between two neighbouring cross-links. But we do not know their shape. From the point of view of phase transitions, the instability described in up eqs. $(13,14)$ is rather special : the order parameter $\rho_{q}$ has singular fluctuations at $T=T_{\mathrm{c}}$ not at one point in $q$ space, but on a whole sphere of radius $q^{*}$.

A similar situation can occur in the solidification of a liquid : however, we know following Landau [6] that the instability is masked by the earlier appearance of a first order transition. Mathematically this is related to the existence of cubic terms $\left(\rho^{3}\right)$ in the free energy. But in our case (at least for the symmetrical case discussed here) we do not have cubic terms, and the Landau theory would predict a second order transition.

To make predictions on the complete structure of the low temperature phase, we need a full analysis of the quartic terms for wave vectors comparable to $q^{*}$. This represents a rather formidable problem, but a similar analysis has been carried out recently for block copolymers [8].

At a more refined level, the situation is rather complex :

a) fluctuation effects tend to restore a first order transition, as shown by Brazowskii [7],

b) however, in certain macromolecular phase transitions, fluctuation effects are reduced, because each chain interacts with a large number of other chains [2]. We do not know yet whether this reduction is present in our problem.

But, on the whole, the present analysis, at the Landau level, should be amply sufficient to interpret a first generation of experiments.

3) As explained in the introduction, both optical and mechanical properties of the microphase are of interest :

a) As regards the transparency, whenever $2 \pi / q^{*}$ is much smaller than an optical wavelength, it should not be very much affected by the transition. Thus, our microphase is probably not a good candidate for the explanation of the cold cataract of the eye, studied by the MIT group [9] : but this question should be investigated in more detail.

b) In cases where the microphase would be produced not in melts, but in solutions, the final product may show a porosity at small (and adjustable) scales.

Acknowledgments. - The author has greatly benefited from discussions with L. Monnerie and L. Leibler.

Appendix. - Estimate OF THE RIGIDITY CONSTANT C. - We consider a chain portion between two consecutive cross-links $L_{1}$ and $L_{2}$, and study it's distortions under a fixed polarization vector $\mathbf{P}$. [We shall assume that each $\mathrm{L}_{i}$ attaches together (symmetrically) one A and one B chain.] Our first simplification amounts to considering the points $\mathrm{L}_{1}$ and $\mathrm{L}_{2}$ as being fixed in space. [In reality, this is true only in an average sense.] Then, if we call $I$ the mid point in space between $L_{1}$ and $L_{2}$, we can say that the microscopic analog of the polarization $\mathbf{p}$ is :

$$
\mathbf{p}=\frac{1}{n} \sum_{i}\left(\mathbf{r}_{i}-\mathbf{r}_{\mathbf{I}}\right) .
$$

For relatively small elongations $\mathbf{p}$, we can assume that the elastic free energy for the chain portion under consideration is then 


$$
\begin{array}{r}
F / \text { portions }=\frac{1}{2} k T\left[\frac{p_{x}^{2}}{\left\langle p_{x}^{2}\right\rangle}+\frac{p_{y}^{2}}{\left\langle p_{y}^{2}\right\rangle}+\frac{p_{z}^{2}}{\left\langle p_{z}^{2}\right\rangle}\right]= \\
=\frac{1}{2} k T \frac{p^{2}}{\left\langle p_{x}^{2}\right\rangle}
\end{array}
$$

where $\left\langle p_{x}^{2}\right\rangle$ is a thermal average, to be computed below. If we now compute the energy per site, we must divide (A2) by the number $n$ of monomers per portion. This gives :

$$
C=1 /\left(n\left\langle p_{x}^{2}\right\rangle\right) .
$$

Thus, all what we need is the thermal average at rest of the quantity $\left\langle p_{x}^{2}\right\rangle$. We obtain this assuming that the portions are ideal. Taking our origin at $\mathrm{L}_{1}$ and denoting by $\mathbf{a}_{i}$ the vector linking $(i)$ and $(i+1)$ we find that

$$
\mathbf{p}=\frac{1}{n} \int_{0}^{n} \mathrm{~d} i(n-i) \mathbf{a}_{i}-\frac{1}{2} \int_{0}^{n} \mathrm{~d} i \mathbf{a}_{i} .
$$

Here $i=0$ corresponds to $\mathrm{L}_{1}$ and $i=n$ corresponds to $L_{2}$. Also we have replaced sums by integrals. Raising (A4) to the square and using the average laws for ideal chains

$$
\left\langle a_{i x} a_{j x}\right\rangle=\delta_{i j} a^{2} / 3
$$

we find

$$
\left\langle p_{x}^{2}\right\rangle=\int_{0}^{n} \mathrm{~d} i\left(\frac{1}{2}-\frac{i}{n}\right)^{2} \frac{a^{2}}{3}=\frac{n a^{2}}{36}
$$

and this, when inserted into (A3), leads directly to eq. (7).

The assumption of ideal chain behaviour is probably correct if, in the initial state, the chains were essentially uncorrelated. But all our treatment of averages is very primitive, and the coefficient in (A5) has only qualitative significance.

\section{References}

[1] FLORY, P., Principles of polymer chemistry (Cornell Un. Press, Ithaca NY) chap. 13.

Huggins, M., J. Chem. Phys. 9 (1941) 440.

[2] The validity of the Flory-Huggins picture for melts is discussed in :

De Gennes, P. G., J. Physique Lett. 38 (1977) L-441.

JoANNY, J. F., Thèse $3^{\mathrm{e}}$ cycle, Université Paris 6 (1978).

[3] A review of $\chi$ parameters and compatibility is given in :

Krause, S., J. Macromol. Sci. (Rev. Macromol. Chem.) C 7 (1972) 251.
[4] Scott, R. L., J. Chem. Phys. 17 (1949) 279. TOMPA, H., Trans. Faraday Soc. 45 (1949) 1143.

[5] Joanny, J. F., Leibler, L., J. Physique Lett. 39 (1978) L-951. De Gennes, P. G., Cours Collège de France (1977).

[6] Landau, L., Lifshitz, I. M., Statistical Physics (Pergamon Press, London).

[7] BrazovskII, V., Soviet Physics J.E.T.P. 41 (1975) 85.

[8] LeIBLER, L., to be published.

[9] BENEDEK, G., Proceedings of the Conference on light scattering, Royal Society London (1978) (to be published). 